\title{
Adaptive SON and Cognitive Smart LPN for 5G Heterogeneous Networks
}

\author{
Songlin Sun • Michel Kadoch • Tiantian Ran
}

Published online: 20 January 2015

(C) Springer Science+Business Media New York 2015

\begin{abstract}
To overcome the challenge of large data demanding in future $5 \mathrm{G}$ cellular networks, heterogeneous networks (HetNets) take advantage of low power nodes (LPNs) to enhance capacity and coverage. This paper aims at 5G HetNets and presents a novel scheme of adaptive self-organization network (SON) by integrating cognitive radio $(\mathrm{CR})$ with inter-cell interference coordination (ICIC). Particularly, we combine the spectrum sensing function from $\mathrm{CR}$ and the radio resource layering function from ICIC. Our work addresses the issues of smart low-power node (SLPN) development, which associates appropriate sectorization with radio resource allocation during the self-organization process. We further develop a Hungarian algorithm based self-organization strategy to improve the SLPN adaptive optimization. Simulation results show that our proposed scheme can achieve considerable gain in terms of throughput and coverage, with extra rewards of high flexibility and low complexity in HetNet SON.
\end{abstract}

Keywords $5 \mathrm{G} \cdot$ HetNets $\cdot \mathrm{SON} \cdot$ Cognitive radio $\cdot$ Smart LPN

S. Sun $(\bowtie) \cdot$ T. Ran

School of Information and Communication Engineering,

Beijing University of Posts and Telecommunications,

Beijing, China

e-mail: slsun@bupt.edu.cn

M. Kadoch

Department of Electrical Engineering, Ecole de Technologie

Superieure, Universite du Quebec, Montreal, Canada

\section{Introduction}

As the $4 \mathrm{G}$ wireless networks are being deployed in the world, the fifth generation $(5 \mathrm{G})$ wireless technologies are emerging into research fields. To meet the large data demanding, heterogeneous network (HetNet) has emerged as a new trend to enhance the capacity/coverage and to save energy consumption for the next generation wireless networks [1]. A heterogeneous network consists of nodes with different transmission powers and coverage areas. High power nodes (HPNs) of large coverage are deployed in a planned way for blanket coverage of urban, suburban, or rural areas. Low power nodes (LPNs) of small coverage aim to complement the HPNs for coverage extension and throughput enhancement. Since it brings the network closer to end users, HetNet can significantly enhance the radio link quality and achieve more efficient spectrum reuse [2-6].

The self-organizing network (SON) plays a critical role in taking advantage of HetNets, as the deployment and operation of a cellular network is an extremely complicated task. SON has been invented to integrate network planning, configuration, and optimization into a unified automatic process requiring minimal manual intervention. In the existing standards, 3GPP Release 8 and 9 specified the most important SON related objectives, including interference control, coverage and capacity optimization (CCO), mobility load balancing, mobility robustness optimization (MRO), and energy saving management for homogeneous topology. In 3GPP Releases 10 and 11, SON for HetNets was recommended for technical requirement study. In the open literature, most existing work 
focused on the SON for homogeneous code-division multiple access (CDMA) and LTE systems [7-11]. There is, however, still a huge gap waiting to be filled regarding the architecture, strategies and algorithms for $5 \mathrm{G}$ HetNet SON.

Different from previous work, this paper develops an adaptive SON scheme for $5 \mathrm{G}$ wireless networks by taking into account cognitive radio, inter-cell interference coordination and cell sectorization. By definition, CR is a radio that can change its transmitter parameters by interacting with environment [12] based on two main characteristics, i.e., cognitive capability and reconfigurability [13]. Our SON scheme mainly focuses on the cognitive capability or spectrum sensing in other words, due to its low infrastructure requirement and easy implementation in smart low-power nodes (SLPNs) [14, 15]. Particularly, we employ spectrum sensing to measure the spectral content for self-configuration and optimization in an SLPN.

Moreover, we also adopt the paradigm of ICIC and make it an inherent part of our adaptive SON scheme. ICIC becomes LTE standard to solve inter-cell intra-frequency interference by dividing each cell into cell center and cell edge and then allocating different subcarriers to subscribers in different locations. Static ICIC can be classified to several genres according to the type of spectrum allocation, including soft frequency reuse (SFR), partial frequency reuse (PFR) [16, 17], etc. Our adaptive SON model improves the canonical PFR to achieve better performance. Canonical PFR splits the bandwidth into two parts: full reuse (FR) part and partial reuse (PR) part. The FR-part is identical to frequency reuse-1 whereas the PR-part is allocated to the cell edge users such that the signals are orthogonal to the neighbor users as well as the FR-part. In our scheme, we split the whole bandwidth of an LPN into several parts rather than two parts. This innovation leads to a perfect match to the self-optimization in SON.

The concept of sectorization is also introduced to our scheme as a new dimension of performance optimization. In [18], the authors observed that higher-order sectorization improves throughput for a fixed number of antennas per cell site, and in this case, inter-cell interference appears more spatially white along with a less performance loss when the spectral efficiency is actually computed. In our adaptive SON scheme, the sectorization order can be set by the algorithm automatically to meet the requirements of various situations.

The remainder of the paper is organized as follows. We first introduce the preliminary of existing SON and then explain the motivation of our proposed 5G adaptive SON in Section 2. We then develop in Section 3 the SLPN spectrum sensing and radio resource management (RRM) schemes with focuses on Hungarian algorithm based self-optimization. Finally, Section 4 presents simulation results to evaluate the performance of our design, followed by Section 5 to conclude the paper.

\section{Adaptive SON architecture}

\subsection{Preliminary}

The most important requirement of $5 \mathrm{G}$ network is the provision of higher system capacity. Large-scale deployment of LPNs becomes a cost-efficient way to realize the foreseen capacity explosion in the near future [19]. SON technology plays a key role in HetNet implementation, as it allows the operators to reduce the complexity of managing cochannel interference and thus save operational costs for all macro and heterogeneous communication entities. It also renders more liberty for powerful optimization strategies to suppress co-channel interference and improve energy efficiency.

By definition, self-organization involves several tasks [20]:

1) self-optimization of radio parameters, which can also be denominated as automatic real-time control of radio parameters;

2) self-healing with the goal of minimizing the impact on network performance caused by failures;

3) self-configuration, which enables automatically deriving sensible initial configuration for network equipment as part of the installation process. Evidently network operation with SON has a huge advantage over the conventional mechanism that relies on human intervention or service tools [21]. In this work, we mainly highlight the self-configuration and optimization functions of SON.

\subsection{Existing SON architecture}

3GPP three entities, including network manager (NM), domain manager (DM) and network element (NE) entities in an SON architecture, as well as the corresponding management reference models for the Evolved Universal Terrestrial Radio Access Network (E-UTRAN) [7]. The NM manages one or more DMs, which further control one or more NEs. Automatic management is the core benefit of SON, which can run in the NM, DM or NE depending on different scenarios.

3GPP LTE-Advanced standards further allow there SON architectures in implementation, i.e., 1) centralized SON, 2) distributed SON, and 3) hybrid SON. In centralized architecture, NM is in charge of the self-organization related execution and coordination. LPNs and the eNB are responsible only for monitoring and execution of the functions, 
whereas the remote operation and management (O\&M) in the NM controls SON related applications. The centralized architecture is able to support sophisticated organization strategies at the cost of increasing time delay and sacrificing adaptive capabilities in real time. In distributed SON, the organization entity runs at the NE, such as eNB or LPNs with $\mathrm{X} 2$ and $\mathrm{S} 1$ interfaces. This feature allows realtime execution and does not require a large bandwidth resource and exchange of data. The distributed SON has an advantage of failure resilience because the outage or malfunction of a SON communication entity will affect only one node [22, 23]. Finally, hybrid SON is introduced to combine the centralized and distributed SON architectures. The hybrid architecture inherits the virtues of its parents, however, has a disadvantage of requiring complex coordination between the centralized and distributed SON functions.

\subsection{G SLPN solution}

In this paper, we employ the distributed SON architecture for self-configuration and self-optimization strategies. Specifically, we identify the LPN self-optimization as the key problem and develop a concept of $5 \mathrm{G}$ SLPN.

LPNs are usually deployed in coverage holes, where users may experience terrible wireless connectivity from macro base stations. However, with the deployment of LPNs, although the received signal-to-interferenceplus-noise ratio (SINR) is high near the LPNs, but it rapidly falls to a level below the original macro BS serving SINR, due to the excessive cross-tier interference.

Given a set of possible LPN locations, we may mitigate the excessive interference by optimizing the configuration of LPNs using iterative optimization methods such as integer programming, simulated annealing, and multi-era genetic programming algorithms. The complexity of system optimization, however, can reach an infeasible level due to the cell densification [24], coverage resolution and indoor-outdoor path-loss variation. Hence, there is a temptation to deploy LPNs without articulated planning. Our proposed adaptive SON scheme becomes a good solution in this sense, as it simply relies on signal processing techniques to improve performance.

In general, we have the following principles on the deployment of SLPNs:

1) mitigating cross-tier interference,

2) maximizing the overall capacity of HetNets,

3) flexibility and adaptability, and

4) self-configuration and self-optimization strategies with low-computational complexity.

\section{SLPN for adaptive SON}

The SLPN solution for $5 \mathrm{G}$ adaptive SON is shown in Fig. 1, with which SLPN can automatically configure and optimize itself in a variety of radio environment with fixed period. Along with the deployment, SLPN starts to sense the environment by CR. Then an optimization module works to improve power allocation and sectorization. Finally antenna angle selection and spectrum allocation find the best tradeoff between capacity and interference.

\subsection{Construction of interference matrix}

We consider the case that an SLPN is deployed in a coverage hole due to the penetration loss of thick building walls or downtown area with a large number of people and cars that have more data demand. The construction of interference matrix serves as the first step of SLPN self-configuration with a goal of reducing its' interference to other nodes. SLPN collects the frequency and power distribution of each angle around itself by spectrum sensing.

Consider any area distinguished by cell ID $g$, which is defined as an interference unit (IU) $g$. Noting that each coverage area by LPN is considered with individual IU while there might be several IUs corresponding to different sectors in a macro-cell. According to CR theory, the received power spectrum at angle $\theta_{i}$ can be established as frequency-angle sensing matrix $\mathbf{A}_{g}$ shown in Fig. 2. Here, $j=(0,1,2, \ldots, J-1)$ and $i=(0,1,2, \ldots, I-1)$ stand for the index of frequency bands and the index of angle divisions respectively; red square suffers high interference from IU $g$, yellow square suffers medium interference, and white square suffers minimum interference.

Spectrum sensing matrix $\mathbf{A}_{g}$ aims at showing IU $g^{\prime} s$ influence on SLPN. However, $\mathbf{A}_{g}$ cannot be used directly and must be extended to single interference matrix $\mathbf{B}_{g}$ by geometrical relationship. Figure 3 gives an interpretation to the relationship between $\mathbf{A}_{g}$ and $\mathbf{B}_{g}$. Using the IU $g$ for example, three sectors form an integrated cell with the

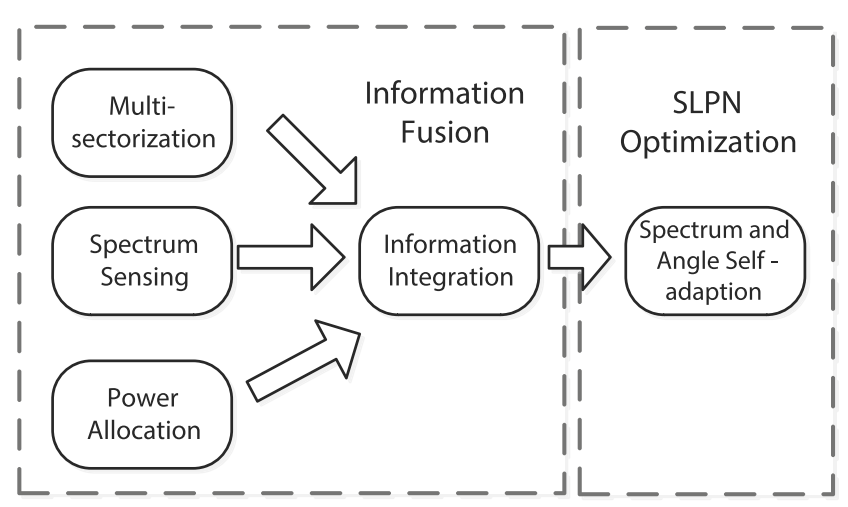

Fig. 1 The flow chart of our proposed 5G SLPN 
Fig. 2 Spectrum sensing matrix $\mathbf{A}_{g}$ by a single power node

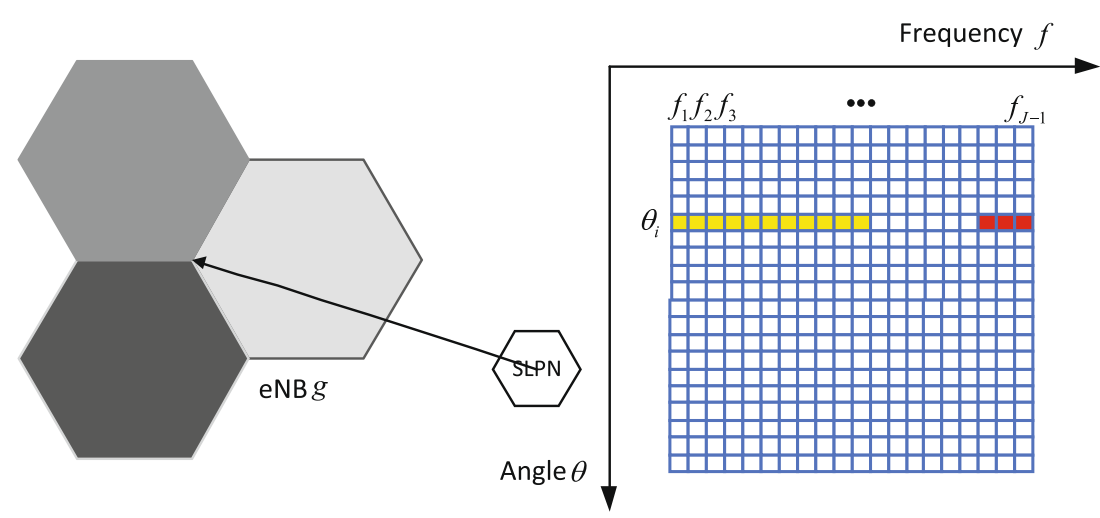

position of each power node known by operator's database. Then, we can easily calculate the cell radius $r$, the distance $d$ between SLPN and the corresponding eNB of IU $g$, and the angle $\alpha$. In details, the extensional angle can also be computed by the following step.

First using cosine formula we have

$l=\sqrt{r^{2}+d^{2}-2 r d \cos \alpha}$.

Then angle $\delta$ is given by

$\delta=\arccos \left(\frac{d^{2}+l^{2}-r^{2}}{2 d l}\right)$.

Similarly,

$\beta=\arcsin \left(\frac{r}{l}\right)$.

Finally, we calculate the extension angle $\beta_{1}$ and $\beta_{2}$ in the following

$\beta_{1}=\beta+\delta$
$\beta_{2}=\beta-\delta$.

The spectrum at angle $\theta$ can be expanded once $\beta_{1}, \beta_{2}$ and $\beta$ are known. For example, the elements at the row $\theta_{i}$ remain unchanged, but the elements from row $\theta_{i}-\beta_{1}$ to row $\theta_{i}+\beta_{2}$ will be added by the row $\theta_{i}$ 's elements. Likewise, the interference to any general LPN can be achieved by Eqs.1-4 in the same way. The single interference matrix $\mathbf{B}_{g}$ is shown in right part of Fig. 3.

Let $\mathbf{Z}$ be the synthetic interference matrix that takes all $G$ power nodes interference matrix $\mathbf{B}_{g}(g=0,1,2, \ldots, G-1)$ into account. Then, $\mathbf{Z}$ is given by the superposition of all $\mathbf{B}_{g}$

$\mathbf{Z}=\sum_{g=0}^{G-1} \mathbf{B}_{g}$.

If there are some continuous angles without power nodes, then the rows of $\mathbf{Z}$ will be empty; If there are some angles with more than one power nodes, then the rows of $\mathbf{Z}$ will be their superposition. Figure 4 demonstrates the formation of matrix $\mathbf{Z}$.

\subsection{Multilayer frequency reuse and power assignment}

We propose in this paper an improved multi-layer cell structure to make full use of the sensed spectrum information and achieve flexibility. It is a common problem for an eNB that edge users are always having to face stronger interference.
Fig. 3 Single interference matrix $\mathbf{B}_{g}$ by a single power node

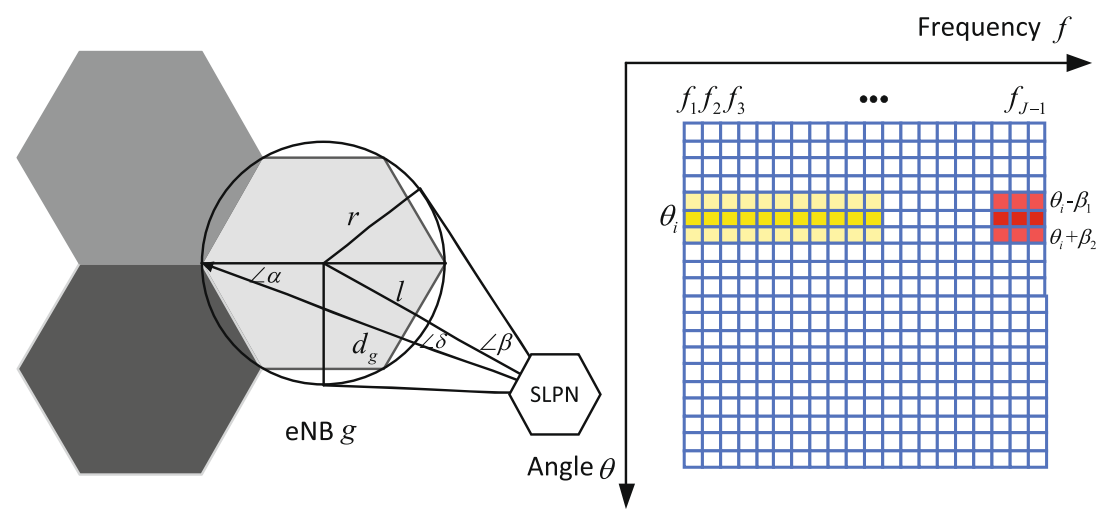


Fig. 4 Synthetic interference matrix $\mathbf{Z}$

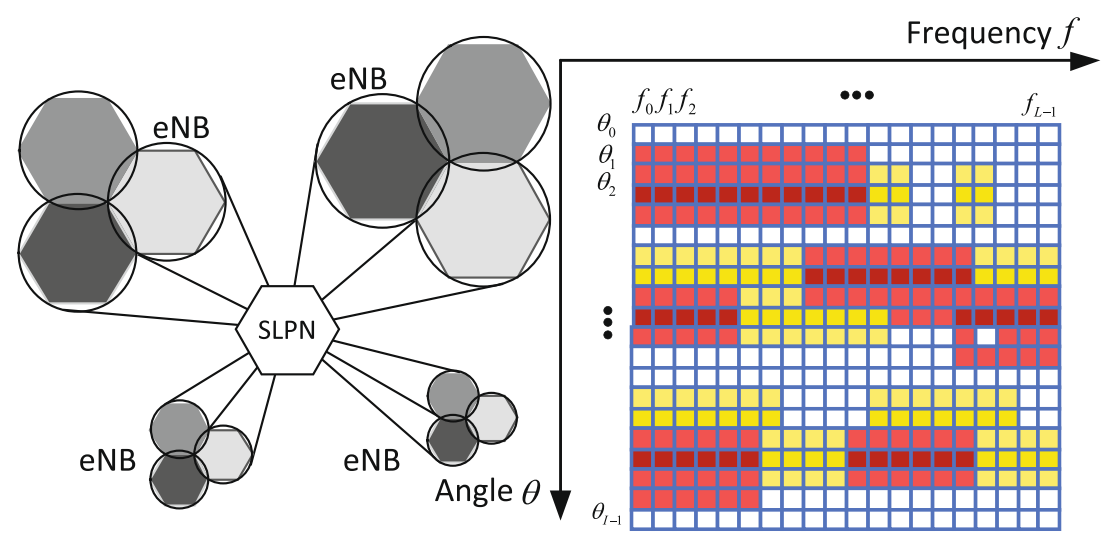

Fig. 5 Layered three-dimension interference matrix $\mathbf{R}$.
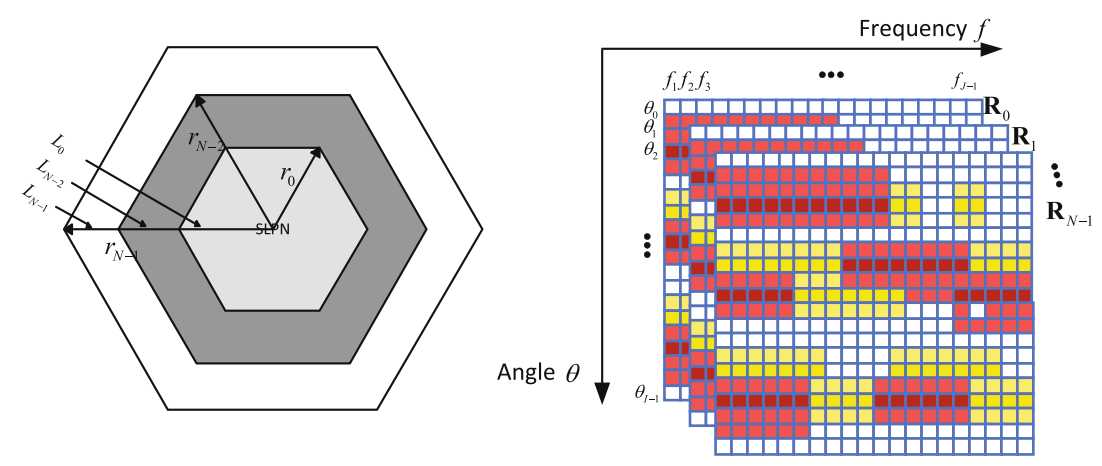

Fig. 6 Multi-sectorized interference matrix $\mathbf{R}^{\prime}$ and the sector $m^{\prime}$ s interference matrix $\mathbf{S}_{m \triangle}$

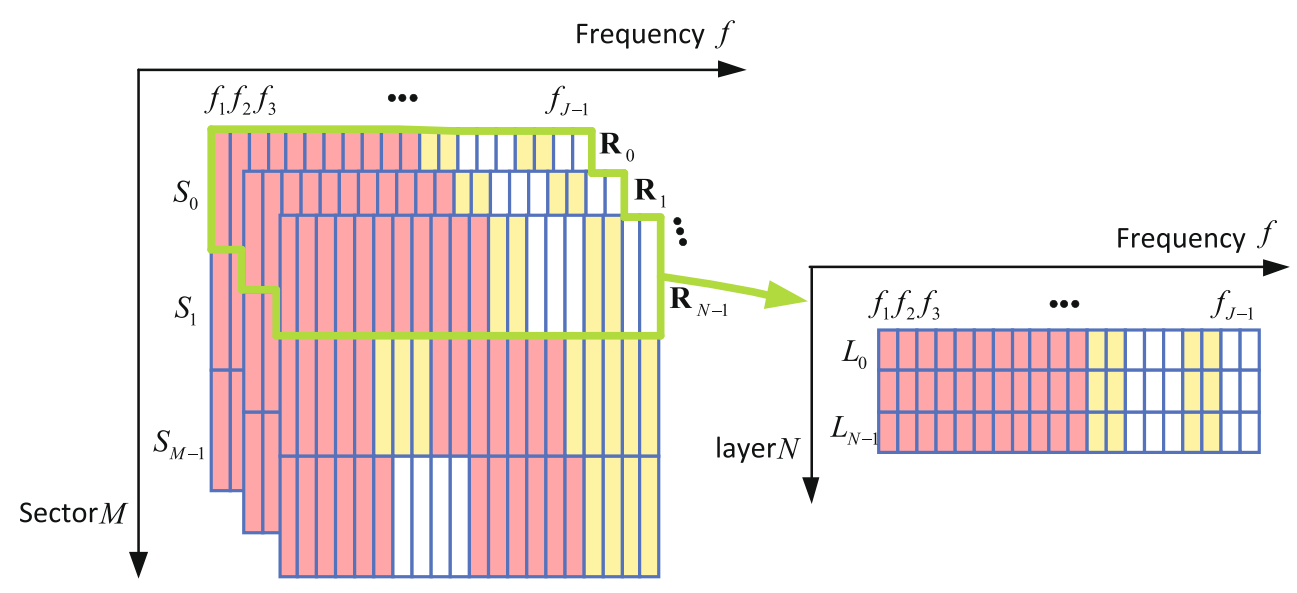

In open literature, there are some static interference coordinating schemes, such as SFR and PFR, which divide the cell into exterior and interior areas with different frequency and power. In our work, we extend the concept of exterior and interior to a multi-layer structure in SLPN. Figure 5 shows that there might be more than 2 layers in our system. As usual, the exterior area always has high power serving edge users whereas the center power is low to decrease interference to other cells. Evidently the frequency selection in different layers is crucial to achieve good performance. 

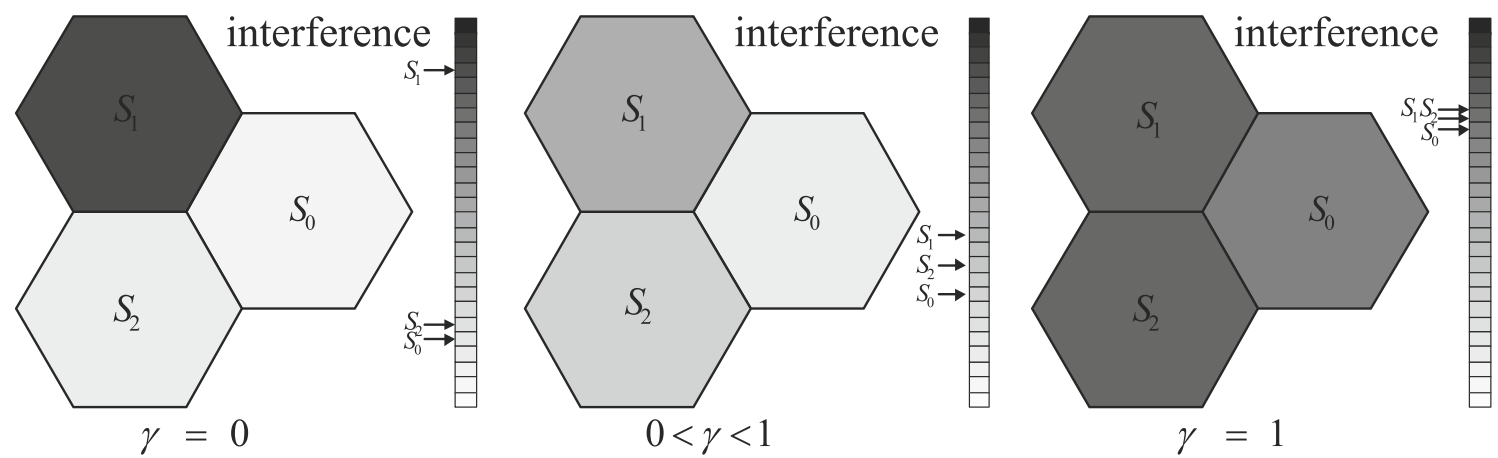

Fig. 7 Trade-off between minimum interference and fairness

Let $P$ be the transmit power of SLPN. To ensure the fairness amongst different layers, the transmit power $P_{n}$ of layer $n$ can be formulated as

$P_{n}=\frac{P r_{n}^{2}}{\sum_{k=0}^{N-1} r_{k}^{2}}$

where $r_{k}$ is the radius of layer $k$. We further define the power node set $\mathbf{G}_{n}$ as $\mathbf{G}_{n}=\left\{g \mid d_{g} \leq l_{g}^{n}\right\}$. where $l_{g}^{n}$ is the SLPN's interference distance of layer $n, d_{g}$ is the distance from SLPN to the corresponding power node of IU $g$. We only

Table 1 Simulation Setup

\begin{tabular}{ll} 
Cellular layout & $\begin{array}{l}\text { Hexagonal grid, 19 cell sites, } 3 \\
\text { sectors per site }\end{array}$ \\
\hline Inter-site distance & 500 \\
System bandwith & $1 \mathrm{GHz}$ \\
Simulation scenario & ITU-UMa \\
Penetration loss & $10 \mathrm{~dB}$ \\
Antenna pattern & $A(\theta)=-$ min $\left[12 \frac{\theta}{\theta_{3 d B}}, A_{m}\right]$, \\
(horizontal) & $\theta_{3 \mathrm{~d} B}=70 \mathrm{degrees}, A_{m}=20 \mathrm{~dB}$ \\
Antenna configuration & Single-Input-Single-Output \\
Channel model & Rayleigh fading model \\
eNB antenna gain & $14 \mathrm{dBi}$ \\
LPN antenna gain & $5 \mathrm{dBi}$ \\
eNB TX power & $46 \mathrm{dBm}$ \\
LPN TX power & $30 \mathrm{dBm}$ \\
Thermal noise density & $-174 \mathrm{dBm} / \mathrm{Hz}$ \\
Downlink interference & Explicit modeling(all links are \\
& simulated) \\
Frequency reuse for eNB & PFR \\
Traffic model & Full buffer \\
User Access strategy & Cell range expansion \\
SLPN sector and layer numbers & $(M=3, N=3)$, if no specification \\
SLPN power allocation & otherwise. \\
& See Eq. 6)
\end{tabular}

consider the interference from SLPN to IU $g$ when $d_{g}<l_{g}^{n}$. Particularly, $l_{g}^{n}$ is given by

$\frac{P_{n}}{P_{\text {loss }}\left(l_{g}^{n}\right) \cdot W_{\text {loss } g}}=P_{\text {lim }}$,

where $P_{\text {loss }}(\cdot)$ is the pass loss function and $W_{\text {loss } g}$ is the wall loss of corresponding power node of IU $g$. $W_{\text {loss } g}$ can be derived by $P_{\text {loss }}\left(d_{g}\right) \cdot W_{\text {loss } g}=P_{T g} / P_{R g}$. where $P_{T g}$ is the transmit power of IU $g$ and the $P_{R g}$ is the received power of IU $g$.

Finally, we define the following to model the interference of our proposed multi-layer frequency reuse of layer $n$.

$\mathbf{R}_{n}=\sum_{g \in \mathbf{G}_{n}} \frac{P_{n}}{P_{T g}} \mathbf{B}_{g}$,

This way, a three-dimension matrix $\mathbf{R}$ takes different layers effect into account and makes a difference from the synthetic interference matrix $\mathbf{Z}$.

\subsection{Multi-sector frequency reuse}

In order to support spatial reuse fully and reasonably,our scheme adopts a multi-sector option that a cell can be divided into several number of sectors. More sectors make sense especially for the case of narrow beams.

We define $r_{i j}^{n}=\left[\mathbf{R}_{n}\right]_{i, j}$, where $n=(0,1,2, \ldots, N-1)$ is the index of different layers. For an SLPN of $N$ layers and $M$ sectors, the orientation of sectors varies with the antenna angle offset $\Delta$ and the circular shift matrix $\mathbf{R}^{\prime}$ is given by

$\left[\mathbf{R}_{n \triangle}^{\prime}\right]_{m, j}=\sum_{i=\Delta}^{\Delta+(m+1) \frac{I}{M}-1} r_{m o d(i, I) j}^{n}=r_{m j}^{\prime n}$.

Figure 6 demonstrates the multi-sectorized version of interference matrix $\mathbf{R}^{\prime}$. For one sector, the $m$ th sector matrix $\mathbf{S}_{m \Delta}$ is defined as $\left[\mathbf{S}_{m \triangle}\right]_{n, j}=r_{m j}^{\prime n \Delta}=s_{n j}^{m \triangle}$. 


\subsection{SLPN optimization algorithm}

Optimization of LPN is vital for $5 \mathrm{G}$ HetNet to increase capacity and coverage. Based on the model established before, we investigate how to choose the best bands for different layers in each sector. The bands must minimize the total interference $C_{m \triangle}$ as much as possible. The frequency selection then becomes an optimization problem, where the bands can be the same at different sectors but not in different layers of one sector.

Correspondingly the optimization can be modeled as a general assignment problem

$$
\begin{array}{ll}
\min & C_{m \triangle}=\sum_{n=0}^{h N-1} \sum_{j=0}^{J-1} f_{n j}^{m \triangle} s_{\bmod (n, N) j}^{m \triangle} \\
\text { s.t. } & \sum_{j=0}^{J-1} f_{n j}^{m \Delta}=1(n=0,1,2, \ldots, N-1) \\
& h N-1 \\
& \sum_{n=0}^{m-1} f_{n j}^{m \Delta}=1 \text { or } 0(j=0,1,2, \ldots, J-1), \\
& f_{n j}^{m \triangle}=0 \text { or } 1 ; s_{n j}^{m \triangle} \geq 0 \\
& m=0,1,2, \ldots, M-1 \\
& h=\max \left(1,\left\lfloor J_{\text {sum }}^{m \Delta} / N\right\rfloor\right)
\end{array}
$$

where $f_{n j}^{m \triangle}$ indicates if the frequency $f_{j}$ is used at layer $n$ of sector $m, h$ is the number of frequency selected by each layer at each sector, $J_{\text {sum }}^{m \triangle}$ is the total frequency number that can be selected by the SLPN. The first constraint means there is at least one frequency unit in each layer at each sector. The second constraint means if the frequency lead into much interference, it may not be selected by the SLPN. $J_{\text {sum }}^{\mathrm{m} \triangle}$ is derived by

$J_{\text {sum }}^{m \triangle}=\operatorname{sum}\left(\mathbf{J}_{\text {thre }}^{m \triangle}\right)$,

where the element of vector $\mathbf{J}_{\text {thre }}^{m \Delta}$ is

$\left[\mathbf{J}_{\text {thre }}^{m \triangle}\right]_{j}= \begin{cases}1 & j \in \mathbf{J}^{m \triangle} \\ 0 & j \notin \mathbf{J}^{m \triangle}\end{cases}$

and the set $\mathbf{J}^{m \triangle}$ is

$\mathbf{J}^{m \Delta}=\left\{j \mid \sum_{n=0}^{N-1} s_{n j}^{m \Delta}<S_{\text {thre }}^{m \triangle}\right\}$.

$\mathbf{J}^{m \triangle}$ implies the SLPN will consider only the frequency that has a total interference less than threshold $S_{\text {thre }}^{m \triangle}$ in one sector.
The optimal solution of Eq. 10 can be achieved by $0-1$ linear programming. When $J \geq N$, Eq. 10 simply turns into

$$
\begin{aligned}
& \min C_{m \triangle}=\sum_{n=0}^{h N-1} \sum_{j=0}^{J-1} f_{n j}^{m \triangle} s_{\bmod (n, N) j}^{m \triangle} \\
& +K \sum_{n=h N}^{J-1} \sum_{j=0}^{J-1} f_{n j}^{m \triangle} \\
& \text { s.t. } \sum_{j=0}^{J-1} f_{n j}^{m \triangle}=1(n=0,1,2, \ldots, J-1) \\
& \sum_{n=0}^{J-1} f_{n j}^{m \Delta}=1(j=0,1,2, \ldots, J-1) \\
& f_{n j}^{m \Delta}=0 \text { or } 1 ; s_{n j}^{m \Delta} \geq 0 \\
& m=0,1,2, \ldots, M-1 \\
& h=\max \left(1,\left\lfloor J_{\text {sum }}^{m \Delta} / N\right\rfloor\right)
\end{aligned}
$$

where $K$ is a penalty factor. Hungarian method can then be used to perform the optimization and obtain the best results for all sectors as shown in Algorithm 1.

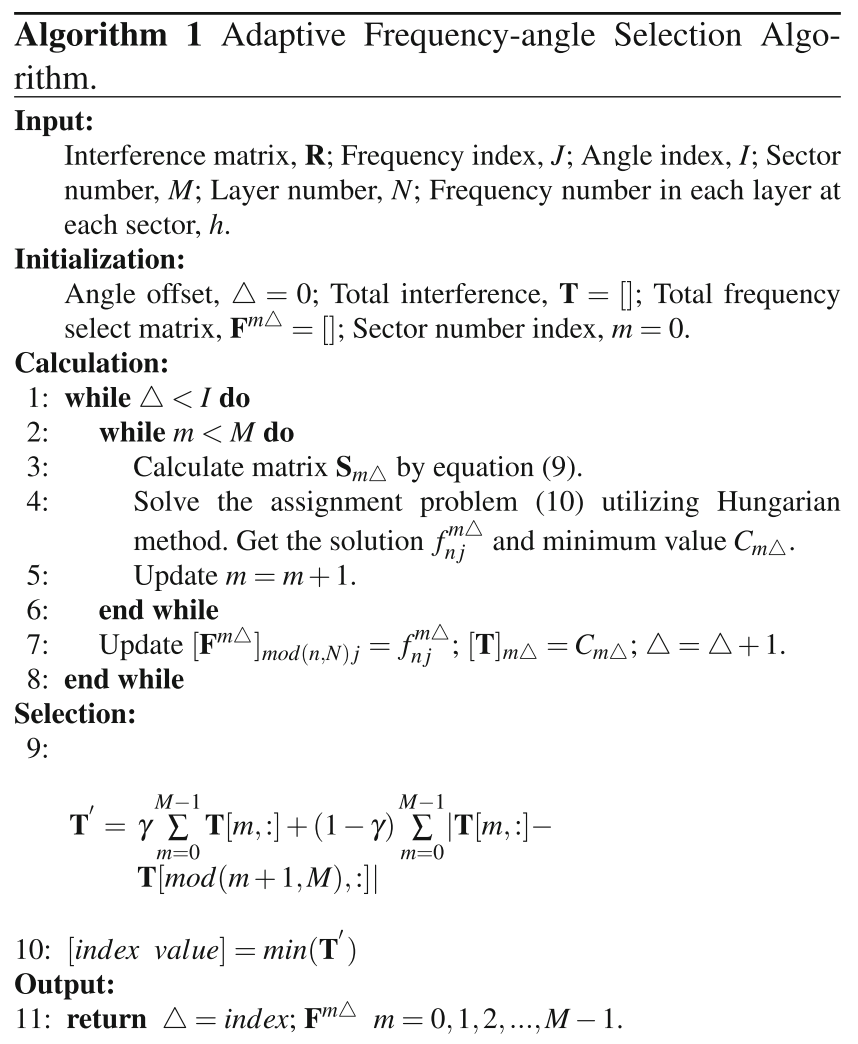

Getting appropriate sectorization angles is not so difficult after getting the frequency allocation for each sector and each angle. The parameter $\gamma$ provides a trade-off between minimum interference and fairness, as shown in Fig. 7. $\gamma=$ 0 is for minimum interference, but may leave a large interference gap or unfairness amongst different sectors; $\gamma=1$ is for complete fairness of sectors, but may cause a considerable increase of overall interference. In practice, we 
Fig. 8 SINR distribution in homogeneous network

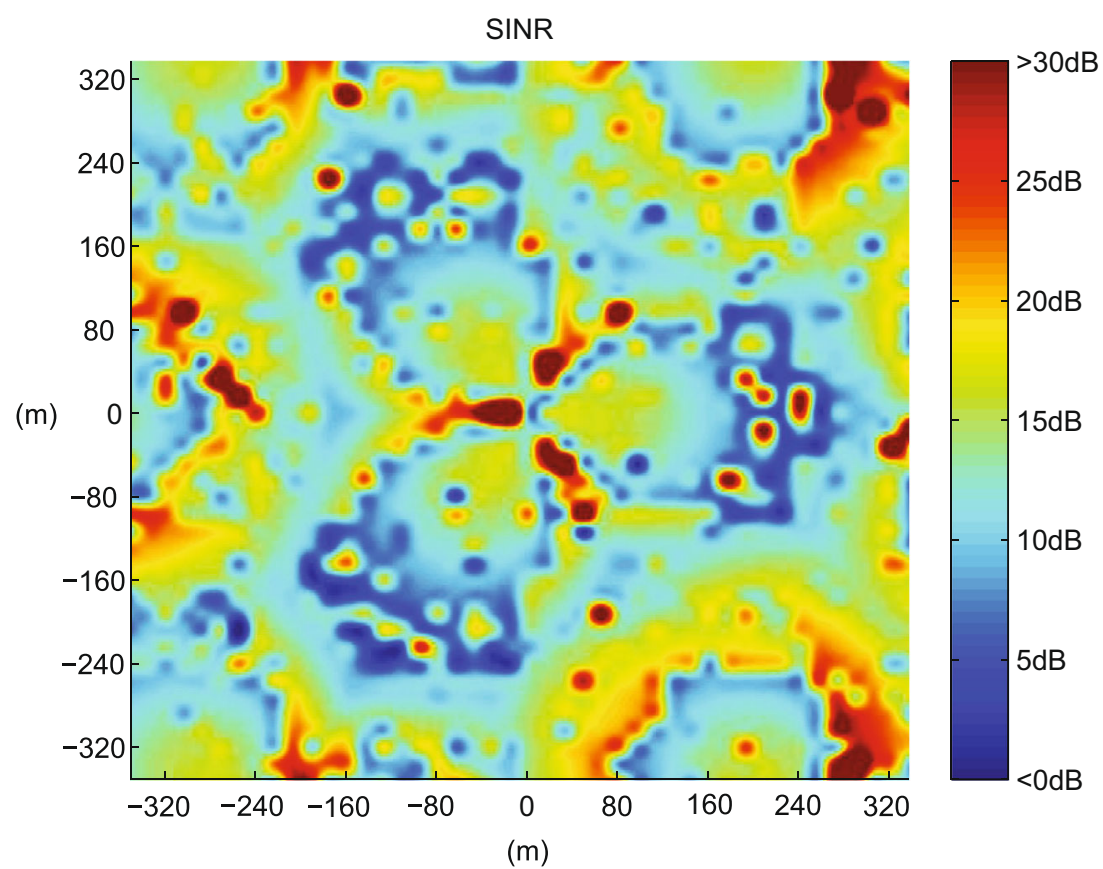

have made a trade-off between minimum interference and fairness by adjusting the parameter $0<\gamma<1$.

\section{Numerical results}

In this section, numerical results are presented to demonstrate the performance of our proposed scheme.The standard scenario of ITU UMa are adopted for all simulations, with UEs uniformly distributed in each hexagonal cell. The main simulation parameters are listed in Table 1.

We first study the original problem of homogeneous network without LPNs as a performance benchmark. This

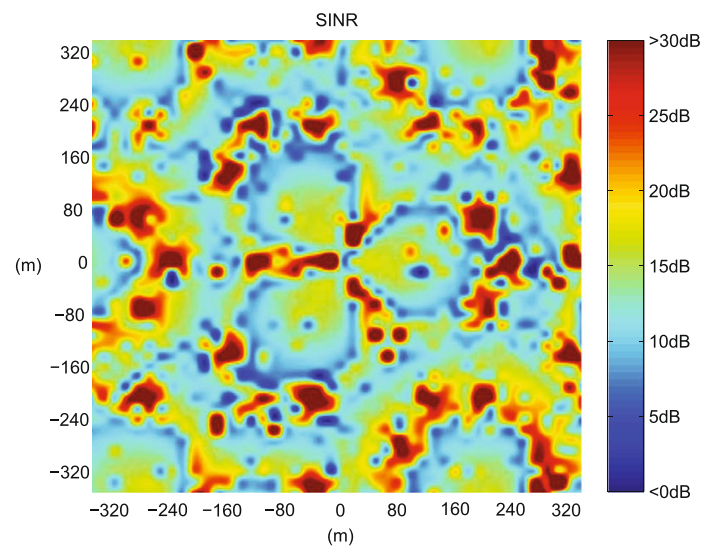

(a) LPN scenario

Fig. 9 SINR distribution in HetNet with LPNs and SLPNs simulation scenario runs a PFR scheme with a $5 \mathrm{~dB}$ threshold and a 0.5 power ratio to distinguish edge users and center users. After that, we compare the performance of our proposed adaptive SON with homogeneous network and HetNet without SON. To testify the advantages of proposed SLPN solutions, SLPNs and LPNs are both deployed in the edge area with coverage holes. Our focus is to demonstrate the advantage of the proposed SLPNs regarding the chosen number of layers and sectors.

Figure 8 illustrates the SINR layout of a homogeneous network, where we can easily spot several coverage holes distributed around the intersection of edge area as well as high buildings. Our simulation study takes into account

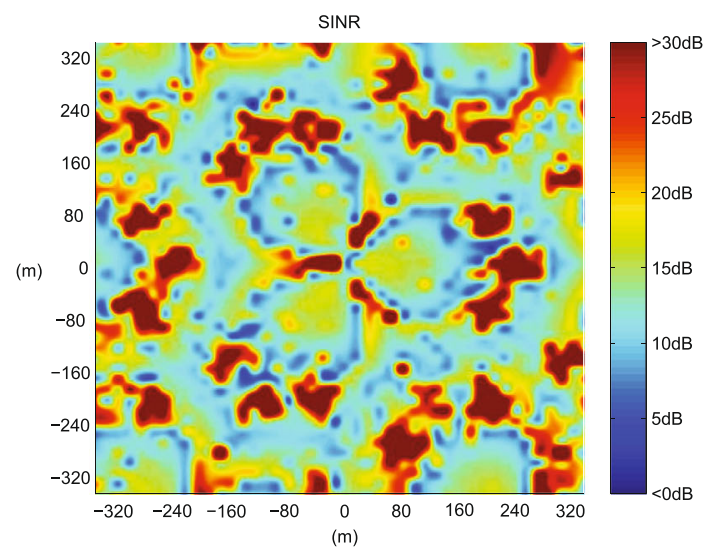

(b) SLPN scenario 
Fig. 10 Coverage probability function of LPNs and SLPNs

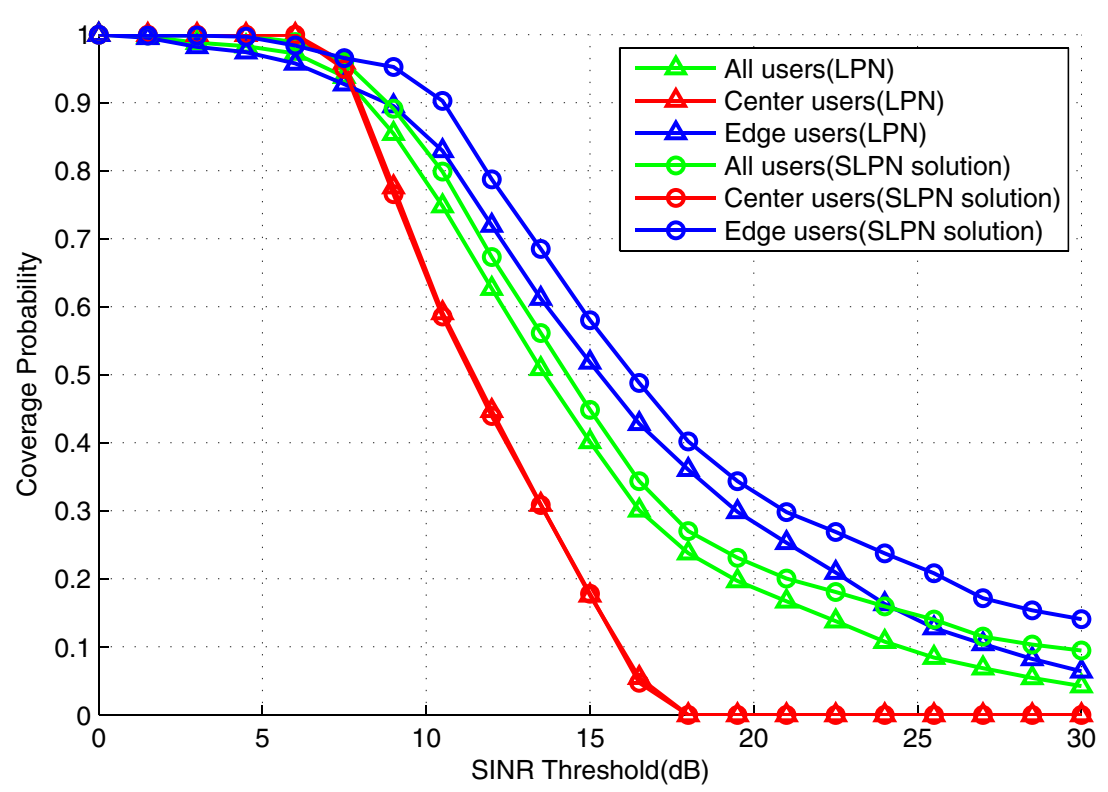

the case that coverage holes may stem from wall loss or deflection caused by buildings. To fill those holes, we add plain LPNs or SLPNs to achieve better performance. The resulting SINR layouts are shown in Figs. 9a and b respectively.

As shown in Fig. 9a, HetNet with plain LPNs indeed improves the coverage performance around coverage holes to some extent. However, this solution fails to improve the overall network performance effectively, as it disregards the interference mitigation in a dynamic environment. In contrast, Fig. $9 \mathrm{~b}$ justifies that SLPN can achieve an SINR layout overcoming the challenge of cross-tier and co-tier interference due to the dynamic algorithm we proposed. The
SLPNs can utilize spectrum sensing of CR technology to help self-configuration and self-optimization, and thus the users attached to those SLPNs can get served with radio resource suffering minimum interference. Though interference to other power nodes always exists in traditional static inter-cell and intra-cell interference coordination, few research in the past concentrated on reducing this effect to the minimum by spectrum sensing, which may be one of the most significant contributions in our scheme.

Our proposed algorithm can not only alleviate the interference in its own cell but also improve the total network performance by reducing interference to other power nodes as much as possible. Figure 10 illustrates different
Fig. 11 Rate probability function of LPNs and SLPNs

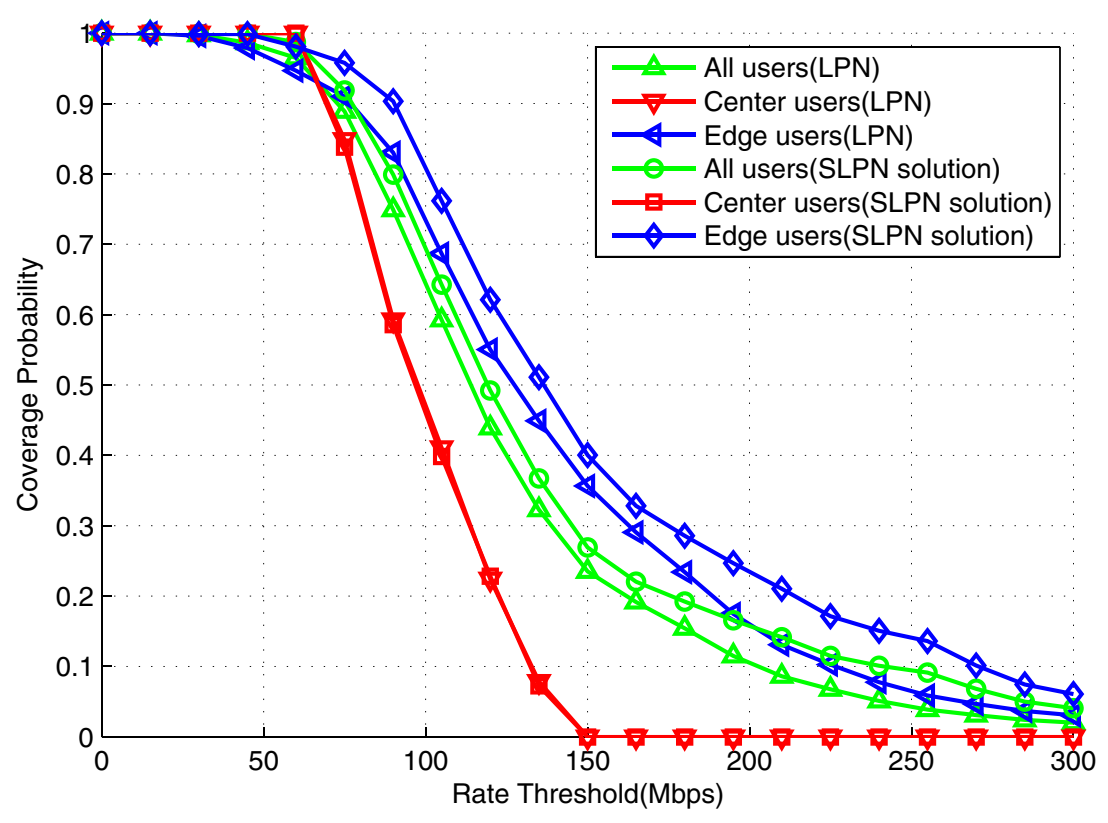


Fig. 12 System throughput of different number of layers and sectors

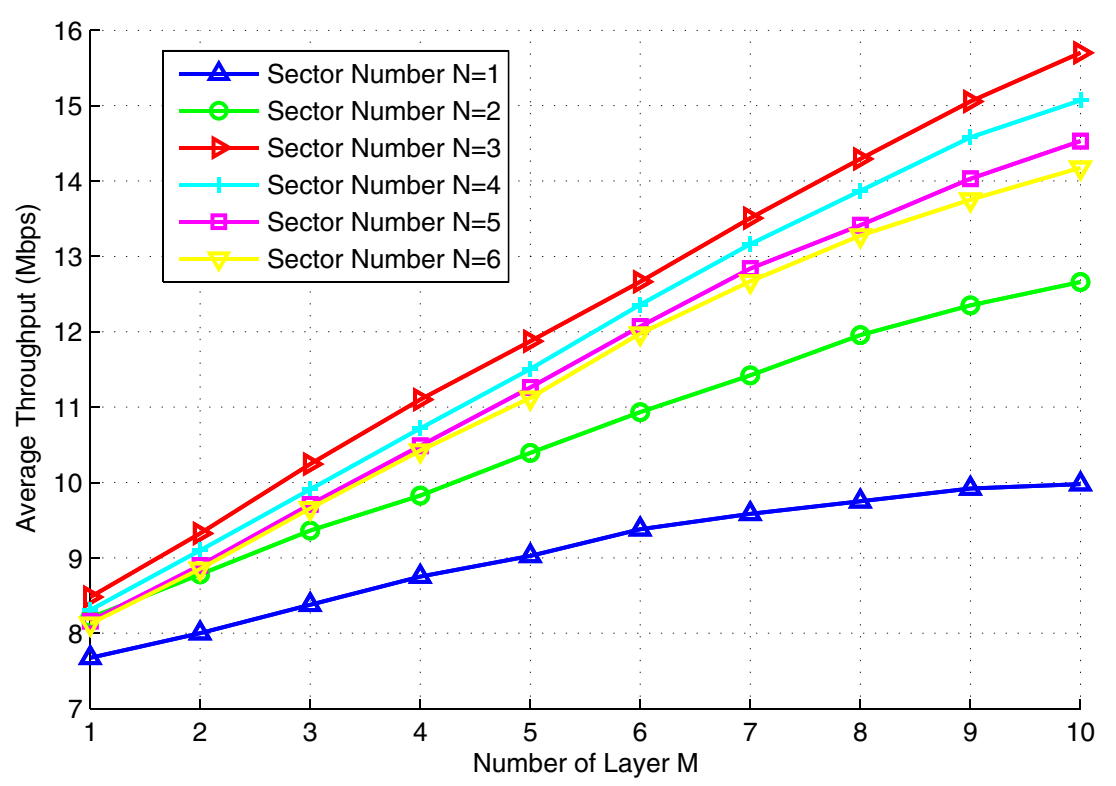

coverage rate between plain LPNs and SLPNs. We can conclude that compared with plain LPNs, SLPNs can improve the throughput of edge users with less impairment to center users and thus the network coverage rate increases significantly. Figure 11 gives the rate probability to further reveal the advantage of our scheme. We can observe that the coverage probability is improved apparently at edge area. On the one hand, SLPN solution introduce less interference to inner users than plain LPN solution. Moreover, the victim users around SLPNs are protected while SLPN can avoid using same RBs with enough cognitive information. As SLPNs are deployed in the edge area, there is no gains for center users, but they can bring more than $2 \mathrm{~dB}$ gains to edge users with coverage probability at 0.9 approximately. With a 10dB SINR threshold, the coverage probability of SLPN solution is $92 \%$ while LPN solution can only cover about $85 \%$ edge users. We can also observe the data rate of edge users with SLPNs is around10Mbps more than normal LPNs.

In our proposed scheme, SLPN can conduct dynamic self-optimization with $M$ sectors and $N$ layers. Accordingly, Fig. 12 demonstrates the impact of the numbers of layers and sectors. With the increasing of layer number $N$, the throughput of SLPN is also growing gradually with more flexible frequency and power allocation strategy. With tri-sector macrocell, the throughput is increased by more than $20 \%$ with 4 layers than 2 layers system. While the layer number is larger than 8 , the growing of throughput is not so obvious, moreover it leads to the growing of computation complexity. With the increase of sector number, the throughput reaches the peak when $M=3$, since $M>3$ might introduce more intra-cell interference.

\section{Conclusion}

This paper develops a new adaptive LPN deployment scheme to improve the throughput and coverage in 5G HetNets. Using our scheme, the SLPNs are able to handle dynamic deployment in a variety of $5 \mathrm{G}$ scenarios. We identify the integration of sectorization and radio resource management as a key problem for SLPNs, and further develop a Hungarian algorithm based self-organization strategy to solve it. Numerical results demonstrate that our scheme can overcome the challenges that HetNet LPNs have to face with low complexity and high flexibility.

\section{References}

1. Hu RQ, Qian Y (2014) An energy efficient and spectrum efficient wireless heterogeneous network framework for $5 \mathrm{G}$ systems. IEEE Commun 52(5):94-101

2. Damnjanovic A, Montojo J, Wei Y, Ji T, Luo T, Vajapeyam M, Yoo T, Song O, Malladi D (2011) A survey on 3gpp heterogeneous networks. IEEE Wirel Commun 18(3):10-21

3. Hu RQ, Qian Y (2013) Heterogeneous cellular networks. John Wiley \& Sons Ltd

4. Li Q, Xu Y, Hu RQ, Qian Y. (2013) Optimal fractional frequency reuse and power control in the heterogeneous wireless networks. IEEE Trans Wirel Commun 12(6):2658-2668

5. Li Q, Hu RQ, Qian Y, Wu G (2013) Intra-cell cooperation and resource allocation in a heterogeneous network with relays. IEEE Trans Veh Technol 62(4):1770-1784

6. Lu K, Qian Y, Guizani M, Chen HH (2008) A Framework for a Distributed Key Management Scheme in Heterogeneous Wireless Sensor Networks. IEEE Trans Wirel Commun 7(2):639-647

7. Peng M, Liang D, Wei Y, Li J, Chen H-H (2013) Selfconfiguration and self-optimization in LTE-advanced heterogeneous networks. IEEE Commun Mag 51(5):36-45 
8. Hu H, Zhang J, Zheng X, Yang Y, Wu P (2010) Self-oonfiguration and self-optimization for LTE networks. IEEE Commun Mag 48(2):94-100

9. Sallent O, Perez-Romero J, Sanchez-Gonzalez J, Agusti R, Diaz-guerra MA, Henche D, Paul D (2011) A roadmap from UMTS optimization to LTE self-optimization. IEEE Commun Mag 49(6):172-182

10. Ramiro J, Hamied K (2006) Self-organizing networks: selfplanning, self-optimization, and self-healing for GSM, UMTS and LTE. IEEE Inf Theory Work:404-408

11. Aliu OG, Imran A, Imran MA, Evans B (2013) A survey of self organisation in future cellular networks. IEEE Commun Surv Tutorials 15(1):336-361

12. (2003) FCC 03-322, Notice of proposed rule making and order

13. Haykin S (2005) Cognitive radio: brain-empowered wireless communications. IEEE JSAC 23(2):201-220

14. Shao YL, Kwang CC, Ying CL, Lin Y (2014) Cognitive radio resource management for future cellular networks. IEEE Wirel Commun 21(1):70-79

15. Gardellin V, Das SK, Lenzini L (2014) Self-coexistence in cellular cognitive radio networks based on the IEEE 802.22 standard. IEEE Wirel Commun 20(2):52-59

16. Huawei (2005) Soft frequency reuse scheme for UTRAN LTE. 3GPP TSG RAN WG1 Meeting \#41, Tdoc (05)0507
17. Godlewski P, Maqbool M, Coupechoux M (2008) Analytical evaluation of various frequency reuse schemes in cellular ofdma networks. In perso.telecom-paristech.fr.

18. Huang H, Trivellato M, Hottinen A, Shafi M, Smith P, Valenzuela $\mathrm{R}$ (2009) Increasing downlink cellular throughput with limited network MIMO coordination. IEEE Trans Wirel Commun 8(6):2983-2989

19. Chandrasekhar V, Andrews J, Gatherer A (2008) Femtocell networks: a survey. IEEE Commun Mag 46(9):59-67

20. Schmelz LC, van den Berg JL, Litjens R, Zetterberg K, Amirijoo M, Spaey K, Balan I, Scully N, Stefanski S (2009) Selforganisation in wireless networks-use cases and their interrelation. Wirel World Res Forum Meet:22

21. Ge X, Han T, Zhang Y, Mao G, Wang C-X, Zhang J, Yang B, Pan $S$ (2014) Spectrum and energy efficiency evaluation of two-tier femtocell networks with partially open channels. IEEE Trans Veh Technol 63(3):1306-1319

22. Bennis M, Perlaza SM, Blasco P, Zhu H, Poor HV (2013) Self-organization in small cell networks a reinforcement learning approach. IEEE Trans Wirel Commun 12(7):3202-3212

23. de Lima CHM, Bennis M, Latva-aho M (2013) Statistical analysis of self-organizing networks with biased cell association and interference avoidance. IEEE Trans Veh Technol 62(5):1950-1961

24. Gelabert X, Legg P, Qvarfordt C (2013) Small cell densification requirements in high capacity future cellular networks. IEEE Commun Work (ICC):1112-1116 